\title{
Use of Z-Codes to Record Social Determinants of Health Among Fee-for-service Medicare Beneficiaries in 2017
}

\author{
William B. Weeks, MD, PhD, MBA' (D), Stacey Y. Cao, MHS ${ }^{2}$, Chris M. Lester, PhD', \\ James $N$. Weinstein, DO, MS ${ }^{1,3}$, and Nancy E. Morden, MD, MPH ${ }^{1,3}$ \\ ${ }^{1}$ Microsoft Research, Microsoft Corporation, Redmond, WA, USA; ${ }^{2}$ Care Journey, Arlington, VA, USA; ${ }^{3} T$ he Dartmouth Institute, Lebanon, NH, USA.
}

J Gen Intern Med 35(3):952-5

DOI: $10.1007 / \mathrm{s} 11606-019-05199-\mathrm{w}$

(C) Society of General Internal Medicine 2019

\section{INTRODUCTION}

Social determinants of health are increasingly recognized as important contributors to individual health, population health, and healthcare spending. ${ }^{1}$ In high-income countries, their inclusion in risk-adjustment models matters. ${ }^{2}$ US adoption of ICD10-CM diagnosis codes in 2015 introduced "Miscellaneous ZCodes" that permit clinicians to record important social determinants or "factors influencing health status." These codes include, for example, education and literacy status or employment status. Identifying and recording such conditions will be key to their future inclusion in risk-adjustment models. We examined Miscellaneous Z-Code use among Medicare feefor-service (FFS) beneficiaries and compared characteristics of beneficiaries with and without Z-Codes. Recognizing the relationship between mental health and social determinants of health, ${ }^{3}$ we explored how Z-Codes were used among beneficiaries with diagnosed psychiatric conditions.

\section{METHODS}

Among Medicare beneficiaries continuously enrolled in FFS Medicare Parts A and B throughout 2017, we identified those with and without a Z-Code diagnosis and compared their demographics, Medicaid enrollment, residential ZIP Code median household income, hierarchical condition category (HCC) scores, and 2017 Medicare Part A and B expenditures. For beneficiaries with a psychiatric diagnosis (one or more ICD10-CM F-Codes), we calculated odds of receiving a Z-Code and, within psychiatric diagnosis categories, examined the prevalence of specific Z-Codes including: education and literacy
(Z55), employment and unemployment (Z56), occupational risk factors (Z57), housing and economic circumstances (Z59), social environment (Z60), upbringing (Z62), and primary social support group (Z63). We used SPSS v25 (2017, Armonk, NY: IBM Corporation) for statistical analyses.

\section{RESULTS}

In 2017, $0.96 \%$ of our cohort had a Z-Code recorded; those with a Z-Code were younger, more likely to be male, black, and Medicaid-enrolled than those without one (Table 1). Compared with those without Z-Codes, beneficiaries with them lived in ZIP Codes with lower median household incomes and had higher HCC scores. Mean per capita Part A and B spending was $\$ 26,852$ (SD \$39,287) among beneficiaries with ZCode diagnoses and $\$ 9,530$ (SD \$22,031) among those without them.

Z-Codes were more commonly recorded among beneficiaries with a psychiatric diagnosis: beneficiaries with alcoholism, drug disorders, and psychotic disorders were most likely to have a Z-Code recorded (Table 2). Among beneficiaries with Z-Code diagnoses, those with dementia had more recorded problems with the social environment, those with substance use and psychotic disorders had more recorded problems with employment and housing and economic circumstances, and those with mood and anxiety disorders had the highest prevalence of primary social support group problems.

\section{DISCUSSION}

In $2017, \mathrm{Z}$-Codes were recorded in less than $1 \%$ of the Medicare FFS population, an astoundingly low proportion of the population (given the well-understood association between socioeconomic status, health, and health services utilization). ${ }^{1}$

Received May 15, 2019

Accepted July 9, 2019

Published online July 19, 2019 
Table 1 Comparison of Medicare Fee-for-service Beneficiaries With and Without Z-Code Diagnoses 2017, Demographics, Illness Burden, Prevalence of Psychiatric Conditions, and Per Capita Medicare A and P Expenditures for Those With and Without a Z-Code

\begin{tabular}{|c|c|c|c|c|}
\hline \multicolumn{2}{|c|}{ Variables } & \multicolumn{2}{|c|}{ Z-Code recorded } & \multirow[t]{2}{*}{$p$ value } \\
\hline & & No & Yes & \\
\hline \multirow{2}{*}{\multicolumn{2}{|c|}{$\begin{array}{l}\text { Number of Medicare FFS beneficiaries } \\
\text { Percent }\end{array}$}} & $30,523,773$ & 297,110 & \\
\hline & & $99.04 \%$ & $0.96 \%$ & \\
\hline \multirow[t]{4}{*}{ Age } & $<18$ & $0.5 \%$ & $0.4 \%$ & \multirow[t]{4}{*}{$p<0.0001$} \\
\hline & $18-45$ & $4.2 \%$ & $14.3 \%$ & \\
\hline & $46-64$ & $12.0 \%$ & $26.0 \%$ & \\
\hline & $65+$ & $83.3 \%$ & $59.3 \%$ & \\
\hline Sex & Male & $54.9 \%$ & $59.9 \%$ & $p<0.0001$ \\
\hline \multirow[t]{4}{*}{ Race } & White & $84.6 \%$ & $80.6 \%$ & \multirow{4}{*}{$p<0.0001$} \\
\hline & Black & $9.3 \%$ & $13.1 \%$ & \\
\hline & Hispanic & $2.0 \%$ & $2.8 \%$ & \\
\hline & Other & $4.1 \%$ & $3.4 \%$ & \\
\hline \multirow{2}{*}{\multicolumn{2}{|c|}{$\begin{array}{l}\text { Medicaid enrolled } \\
\text { Median household income }(\$)\end{array}$}} & $18.4 \%$ & $43.3 \%$ & \multirow{3}{*}{$\begin{array}{l}p<0.0001 \\
p<0.0001\end{array}$} \\
\hline & & 61,679 & 58,512 & \\
\hline \multicolumn{2}{|c|}{ STDEV } & 25,848 & 24,295 & \\
\hline \multicolumn{2}{|c|}{$\begin{array}{l}\text { Hierarchical condition category score } \\
\text { STDEY }\end{array}$} & 1.04 & 1.35 & \multirow[t]{2}{*}{$p<0.0001$} \\
\hline \multicolumn{2}{|c|}{$\begin{array}{l}\text { STDEV } \\
2017 \text { per canita Medicare Part }\end{array}$} & 1.03 & 1.28 & \\
\hline \multirow{2}{*}{\multicolumn{2}{|c|}{$\mathrm{A}$ and $\mathrm{B}$ expenditures $(\$)$}} & 9350 & 26,852 & \multirow[t]{3}{*}{$p<0.0001$} \\
\hline & & & & \\
\hline \multicolumn{2}{|c|}{ STDEV } & 22,031 & 39,287 & \\
\hline
\end{tabular}

Psychiatric conditions are measured by presence of ICD-10-CM diagnostic F-codes. STDEV means standard deviation. Median household income is measured at the beneficiary's residential ZIP Code. $p$ value tests for statistical difference across columns using Student's t test for continuous variables and the chi-square test for categorical variables

This suggests Z-Codes were not being used nearly as often as would likely be appropriate. While beneficiaries with Z-Codes were younger, were sicker, and had annual medical expenditures almost threefold that of beneficiaries without them, the low rate of Z-Code use suggests they constitute a specific, but not sensitive, measure of socioeconomic need. Z-Codes were more commonly recorded among beneficiaries with psychiatric diagnoses; this may represent bias by providers recording these codes. Mental health professionals have a long used a multi-axial diagnostic framework that includes an evaluation of psychosocial and environmental factors that affect global functioning; they may simply be more accustomed to recording social determinants of health.

Our study is limited by its cross-sectional nature and reliance on administrative data. The observed patterns reflect clinician adoption of codes shortly after their introduction; current practice may differ.
Nonetheless, the observed patterns align with expectations: recorded social determinants correlate with indicators of poverty and behavioral health conditions. While we do not know the true prevalence of conditions codified by Z-Codes or the extent to which these conditions are explored by clinicians, the observed prevalence undoubtedly misses the majority of cases. Using natural language processing to extract and code data from electronic medical records ${ }^{4}$ may facilitate recording and categorization of social determinants of health and accelerate their use in population health management and value-based payment models. ${ }^{5}$ More importantly, routinely capturing that information might signal specific, important needs of these patients to care managers, care teams, payers, and policy makers who aim to leverage public resources to optimize healthcare delivery value and improve the population's health. 


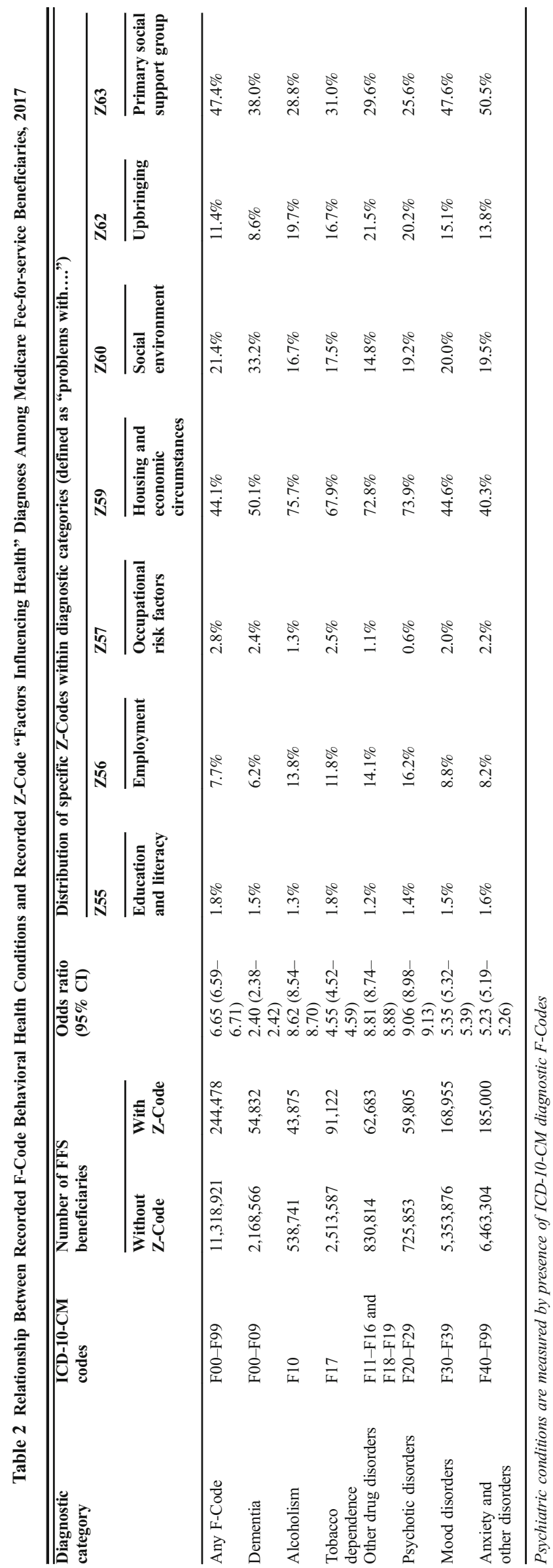


Corresponding Author: William B. Weeks, MD, PhD, MBA; Microsoft Research, Microsoft Corporation, Redmond, WA, USA (e-mail: william. weeks@microsoft.com).

\section{Compliance with Ethical Standards:}

CareJourney has IRB approval ("Understanding the drivers of ACO success in achieving low cost and high quality care"; Solutions IRB Study No. 2017/08/15) to study health service utilization using CMS data (CMS DUA 51593) through their Virtual Research Data Center.

Conflict of Interest: The authors declare that they do not have a conflict of interest.

\section{REFERENCES}

1. Fink DS, Keyes KM, Cerda M. Social Determinants of Population Health: A Systems Sciences Approach. Current epidemiology reports 2016;3:98105.
2. Stringhini S, Carmeli C, Jokela M, et al. Socioeconomic status and the 25 x 25 risk factors as determinants of premature mortality: a multicohort study and meta-analysis of 1.7 million men and women. Lancet (London, England) 2017;389:1229-37.

3. Allen J, Balfour R, Bell R, Marmot M. Social determinants of mental health. International review of psychiatry (Abingdon, England) 2014;26:392-407.

4. Navathe AS, Zhong F, Lei VJ, et al. Hospital Readmission and Social Risk Factors Identified from Physician Notes. Health Serv Res 2018;53:111036.

5. Gottlieb L, Tobey R, Cantor J, Hessler D, Adler NE. Integrating Social And Medical Data To Improve Population Health: Opportunities And Barriers. Health affairs (Project Hope) 2016;35:2116-23.

Publisher's Note Springer Nature remains neutral with regard to jurisdictional claims in published maps and institutional affiliations. 